\title{
Actin and Type I Collagen Propeptide Distribution in the Developing Chick Cornea
}

\author{
Claire Gealy, ${ }^{1}$ Anthony J. Hayes, ${ }^{1}$ Rebecca Buckwell, ${ }^{1}$ Robert D. Young, ${ }^{2}$ Bruce Caterson, ${ }^{1}$ \\ Andrew J. Quantock, ${ }^{2}$ and James R. Ralphs ${ }^{1}$
}

Purpose. To determine the organization of actin filaments and distribution of type I procollagen during the development of the chick corneal stroma.

Methods. Embryonic chicken corneas of ages 6 to 18 days and 18 days posthatch were cryosectioned and fluorescently labeled for filamentous actin with phalloidin and for the $\mathrm{N}$-and C-terminal propeptides of type I procollagen with specific monoclonal antibodies. Tissue sections were examined by fluorescence and confocal microscopy.

Results. Prominent actin filament bundles were present at all embryonic stages, arranged in orthogonal arrays. Type I collagen propeptides were also present, with the C-propeptide visible as small foci, often associated with the actin label. The $\mathrm{N}$-propeptide was also detected in the stromal matrix, especially in Bowman's layer. Actin filaments were also prominent in the corneal epithelium, along with collagen propeptide labeling, up to embryonic day14.

Conclusions. Actin filament bundles are abundant in the stroma, presumably in the keratocytes of the developing chick cornea, and are arranged in an orthogonal manner suggesting a possible role in cell and matrix organization in this tissue. Filament bundles appear to be closely associated with the foci of type I procollagen label, suggesting a possible association between the actin cytoskeleton and the trafficking of collagen. The presence of the N-propeptide of type I collagen in the extracellular matrix and the restricted distribution of the $\mathrm{C}$ propeptide suggest differential processing of these molecules after secretion. The persistence of the N-propeptide implies a role in development, possibly in association with control of collagen fibril diameter and spacing. (Invest Ophthalmol Vis Sci. 2009;50:1653-1658) DOI:10.1167/iovs.08-2554

$\mathrm{T}$ he cornea is an extraordinary structure, with its properties of transparency and precise shape enabling it to perform its crucial function as the principal refractive component of the eye. It is a predominantly collagenous tissue, with most of the tissue thickness composed of the stroma, which consists of a large number of collagenous lamellae and collagen-synthesizing fibroblasts. Morphogenesis of the chick cornea is well

From the ${ }^{1}$ Connective Tissue Biology Laboratories, School of Biosciences and the ${ }^{2}$ Structural Biophysics Group, School of Optometry and Vision Sciences, Cardiff University, Cardiff, United Kingdom.

Supported by the UK Biotechnology and Biological Sciences Research Council Grant BBS/B/10994 (AJQ, JRR, BC).

Submitted for publication July 11, 2008; revised November 6 and 20, 2008; accepted February 4, 2009.

Disclosure: C. Gealy, None; A.J. Hayes, None; R. Buckwell, None; R.D. Young, None; B. Caterson, None; A.J. Quantock, None; J.R. Ralphs, None

The publication costs of this article were defrayed in part by page charge payment. This article must therefore be marked "advertisement" in accordance with 18 U.S.C. $\$ 1734$ solely to indicate this fact.

Corresponding author: James R. Ralphs, Connective Tissue Biology Laboratories, School of Biosciences, Cardiff University, Biomedical Sciences Building, Museum Avenue, Cardiff, United Kingdom CF64 2QR; ralphs@cf.ac.uk. characterized and consists of a sequence of developmental events resulting in the formation of a fully functional transparent tissue. ${ }^{1-6}$ Briefly, at embryonic day (E) 4 , the corneal epithelium derived from the ectoderm deposits a loose matrix of collagen fibrils that form the primary stroma. This primary stroma is believed to act as a template for deposition of the mature, secondary stroma. By E5 of development, mesenchymally derived cells from the neural crest migrate posterior to the primary stroma to form the corneal endothelium. By E6, the primary stroma is invaded by the presumptive corneal fibroblasts, which synthesize and deposit components of the mature, secondary stroma. By E14 the stromal fibroblasts reach adult numbers, and between E14 and E18 ultrastructural changes occur in matrix architecture that contribute to transparency. ${ }^{2,7,8}$ Transparency and biomechanical stability are dependent on the precise organization of collagen fibrils within the corneal stroma. Fibrils are oriented parallel to each other within a lamella but at large angles to each other in adjacent lamellae, resulting in a stacked, lamellar arrangement of collagen fibrils. Therefore, the process by which corneal fibroblasts deposit collagen fibrils, which are regularly spaced and of thin diameter, ${ }^{9-11}$ is crucial to the acquisition of transparency and tissue strength, as is the orientation of the cells. The study that has been paramount in understanding the mechanism involved in collagen fibril deposition is that of Birk and Trelstad. ${ }^{7}$ Briefly, collagen fibrillogenesis was proposed to initially occur within small surface recesses on corneal fibroblasts aligned along the cell axis, which proceed to fuse laterally to form collagen bundles and then lamellae. Cells use this structural arrangement to deposit oriented collagen fibrils and to contribute with others to the formation of oriented lamellae.

In connective tissues, cell orientation and cell-cell interactions play key roles in generating and maintaining matrix architecture. The corneal stroma shares some structural characteristics with the annulus fibrosus of the intervertebral disc in this regard. The annulus fibrosus has a lamellar organization of oriented collagen fibers, ${ }^{12,13}$ with fibrils within a lamella aligned parallel to each other and fibrils in consecutive lamellae aligned at angles to those of adjacent lamellae. The cell orientation process is driven by components of the cytoskeleton, namely actin stress fibers, and by cell-cell interactions through adherens junctions (vinculin and $\mathrm{N}$-cadherin) and gap junctions (connexin 43). ${ }^{14}$ In the annulus fibrosus, stress fibers run parallel to each other in cells within a cell sheet, ensuring that all cells have the same orientation in a cell layer. In the developing chick corneal stroma, mechanisms governing early matrix orientation are unclear. It has been thought, however, that keratocyte precursors migrate to the highly organized primary corneal stroma and become oriented to $\mathrm{it}^{15,16}$ and then deposit the oriented secondary stroma. Cell movement and shape are controlled by the cytoskeleton. It is clear that actin plays a role in determining the orientation of cells in the intervertebral $\operatorname{disc}^{14}$; thus, it is important to determine the role of actin in keratocyte behavior in the corneal stroma.

It is well documented that actin stress fibers containing $\alpha$-smooth muscle actin occur in myofibroblasts in wounded corneas and in corneal keratocytes cultured in vitro. ${ }^{17,18}$ How- 
ever, quiescent corneal keratocytes in vivo do not express stress fibers but, rather, show a cortical distribution of actin associated with the cell membrane. ${ }^{18}$ Corneal epithelial cells also express prominent actin filament bundles. ${ }^{19,20}$ A recent study using multiphoton microscopy showed that phalloidinstained keratocytes in developing chick corneal stroma were in alignment and rotated with second harmonic signals generated by collagen. ${ }^{21}$ Recent studies on tendon development have shown the presence of a small structure, the fibripositor, at the surface of the tendon fibroblast associated with deposition of the earliest oriented collagen fibrils. ${ }^{22}$ The formation and actions of these structures are proposed to be actin dependent. $^{23,24}$

Fibrillar collagen is synthesized intracellularly as procollagen, with $\mathrm{N}$ - and $\mathrm{C}$ - terminal globular procollagen domains that are cleaved on secretion. Corneal epithelial cells have been reported to synthesize procollagen at early stages of development (approximately E5-E12), in association with the formation of the primary stroma, whereas keratocytes partake in synthesis over the entire corneal embryonic period. ${ }^{16,25}$

In this article we describe the distribution of actin filaments stained with phalloidin, and type I procollagen labeled with antibodies to the $\mathrm{N}$ - and $\mathrm{C}$-propeptides, in the developing chick cornea. We show the presence of actin filament bundles in corneal epithelium and stromal keratocytes, with a highly ordered orthogonal array of filament bundles in the developing stroma. We further show the presence of type I collagen C-propeptide in small foci, possibly secretory vesicles, in association with actin filament bundles in keratocytes at all embryonic stages and in epithelial cells until E12. Taken together, the findings suggest a role for actin in organized matrix secretion in primary and secondary stroma. Finally, we show the persistence of the N-terminal propeptide in the extracellular matrix, suggesting possible roles related to matrix assembly.

\section{Materials ANd Methods}

Fertilized White Leghorn chicken eggs (Henry Stewart, Lincolnshire, UK) were incubated at $38^{\circ} \mathrm{C}$ in a humidified chamber until E6, E8, E10, E12, E14, E16, and E18. In addition, corneas were obtained from 18-day posthatch chickens. All animals were killed in accordance with the ARVO Statement for Use of Animals for Ophthalmic and Vision Research. Chick embryonic corneas (E10-E18; day 18) or whole chick embryonic eyes (E6 and E8) were frozen unfixed on dry ice in OCT embedding medium (Tissue-Tek; Sakura Finetek Europe, Leiden, The Netherlands), and 10- or 20- $\mu \mathrm{m}$ cryosections were cut in transverse or tangential planes. For labeling procedures, cryosections were rehydrated in PBST (0.05 M phosphate-buffered saline, $\mathrm{pH} 7.3$, containing $0.1 \%$ Tween-20; also used as labeling reagent diluent) and were blocked with $5 \%$ normal goat serum. For the detection of filamentous actin, cryosections were directly labeled with Alexa Fluor 488-conjugated phalloidin $(0.15 \mu \mathrm{M}$; Invitrogen-Molecular Probes, Carlsbad, CA) for 45 minutes at room temperature, washed twice in PBST, and mounted in mountant containing DAPI as a nuclear counterstain (Vectashield; Vector Laboratories, Burlingame, CA). Immunohistochemical labeling was performed by indirect immunofluorescence with primary antibodies to type I procollagen; for controls, primary antibodies were omitted or substituted with nonimmune mouse immunoglobulins (10 $\mu \mathrm{g} / \mathrm{mL}$; Dako, Carpinteria, CA). Sections were incubated for 1 hour with monoclonal antibodies M-38 $(10 \mu \mathrm{g} / \mathrm{mL}$; recognizing C terminus type 1 procollagen $\left.{ }^{26}\right)$ or SP1.D8 $(10 \mu \mathrm{g} / \mathrm{mL}$; recognizing N-terminus type I procollagen ${ }^{27}$ ), obtained from the Developmental Studies Hybridoma Bank (developed under the auspices of the National Institute of Child Health and Human Development and maintained by the Department of Biological Sciences at the University of Iowa, Iowa City, IA). Sections were washed in PBST and detected by incubation with Alexa Fluor $594 \mathrm{~F}\left(\mathrm{ab}^{\prime}\right)_{2}$ fragments of goat anti-mouse IgG $(5 \mu \mathrm{g} / \mathrm{mL}$; Invitrogen-Molecular Probes) for 1 hour. After further washing, they were mounted as described. For dual labeling, sections were incubated sequentially with Alexa Fluor 488-phalloidin, antibodies to procollagen, and the AlexaFluor 594 goat anti-mouse IgG secondary antibody at a concentration of $5 \mu \mathrm{g} / \mathrm{mL}$. Results were analyzed under a microscope (BX61; Olympus, Tokyo, Japan) and an F-view digital camera or under a confocal microscope (SP2 AOBS; Leica, Wetzlar, Germany). Control sections showed no autofluorescence or nonspecific label of any kind in wide-field or confocal microscopy.

\section{Results}

\section{Actin Filaments in the Developing Chick Cornea}

Actin was detected with phalloidin at each stage investigated (E6-E18, 18 days posthatch; Fig. 1). At E6 (Figs. 1a, 1b), actin label was prominent in the corneal epithelium, lens, and stroma. Stromal label was in the form of filament bundles, with an indication of orthogonal organization through the tissue. Filament bundles were clearly visible in the plane of the section, with others running at right angles to them that were visible as spots of bright label, where they were transversely sectioned. At E8 and E10, the stromal fibroblasts had developed more extensive and prominent actin filament bundles (Figs. 1c, 1d); corneal epithelial cells continued to label strongly for actin (Fig. 2). This pattern of stromal and epithelial labeling persisted through E12 to E18 (Figs. 1e, 1f). Confocal microscopy of tangential sections demonstrated the orthogonal nature of actin fiber organization. In the example shown (Fig. 1g, E16) an extensive three-dimensional orthogonal actin array is clearly visible, with actin filament bundles running in $X$ and $Y$ planes relative to the picture plane (streaks) and in the $Z$-plane (confocal section series; dots). Rotating three-dimensional models reveal the dots to be $Z$-plane filament bundles (Fig. $1 \mathrm{~h}$; see also animation movies in the Supplementary Data, http://www.iovs. org/cgi/content/full/50/4/1653/DC1). At E18, actin filament bundles were still present though there was also a more generalized staining of the cells (Fig. 1i). Actin label was also present in a day 18 posthatch cornea, though filament bundles were less obvious (Fig. 1j).

\section{Type I Procollagen Distribution and Relation to Actin Distribution}

The two antibodies used to determine type I procollagen distribution, M-38 and SP1.D8, bind to the C-terminus and N-terminus of type I procollagen, respectively. At E6, M-38 label was present prominently in association with epithelial cells and more weakly with keratocytes (Figs. 2a, 2b). The label, in the form of focal aggregates, was judged to be intracellular because it was generally close to the nuclei and/or cytoskeletal filaments (Fig. 2i). SP1.D8 labeled more extensively (Figs. 2c, 2d); it resembled the M-38 label in association with epithelial cells and keratocytes and labeled the stromal extracellular matrix with Bowman's layer particularly prominently. The stromal patterns of M-38 and SP1.D8 immunoreactivity persisted throughout the period studied (e.g., Figs. 2e-h). In the epithelium, procollagen label became sparse at E12 and was absent from E14 onward.

Dual-label studies showed a possible association between some of the type I procollagen label and the actin cytoskeleton. Low-power studies showed the extensive tissue-wide distribution of both (Figs. 2g, 2h); high-power studies showed an apparent association between procollagen and actin filament bundles (Figs. 2i-k). At early stages, actin filament bundles were associated with punctate type I procollagen label, as identified using antibody M-38 in the epithelium and stroma (Figs. 2i, 2j). This continued in the epithelium until procollagen disappeared at E14 but was maintained in the stroma throughout the em- 
Figure 1. Phalloidin-labeled filamentous actin in developing chicken cornea; nuclei are counterstained blue with DAPI; (g) and (h) are derived from confocal section series; the remainder are wide-field fluorescence images. (a) E6 embryo. Low magnification showing bright label in epithelium (arrow) and lens (asterisk) with label clearly present in the corneal stroma (s). The epithelium has separated from the stroma in sectioning. (b) High magnification of corneal stroma. Keratocytes contain prominent actin filament bundles showing evidence of orthogonal orientation. Some run longitudinally in the section plane (arrows), and others are sectioned transversely and appear as dots (arrowheads). (c, d) Corneal stroma in E8 and E10 embryos. Stromal fibroblasts contain extensive networks of actin filament bundles. (e) Transverse section of E14 corneal stroma. Cells contain prominent actin filament bundles; arrow points to region displaying prominent streaks of actin running longitudinally relative to the section plane, and adjacent spots of label representing filament bundles sectioned transversely. (f) Transverse section of E16 cornea. In addition to the actin filament bundles shown in (e), this image contains short lengths of actin filament bundles running at right angles to both (i.e., in the antero-posterior plane of the cornea; arrows). (g) Projection prepared from confocal section series of E16 stroma sectioned tangentially. Orthogonal arrays of actin filament bundles are clearly visible in the $X$ and $Y$ directions relative to the image plane. Spots of label suggest filaments running in
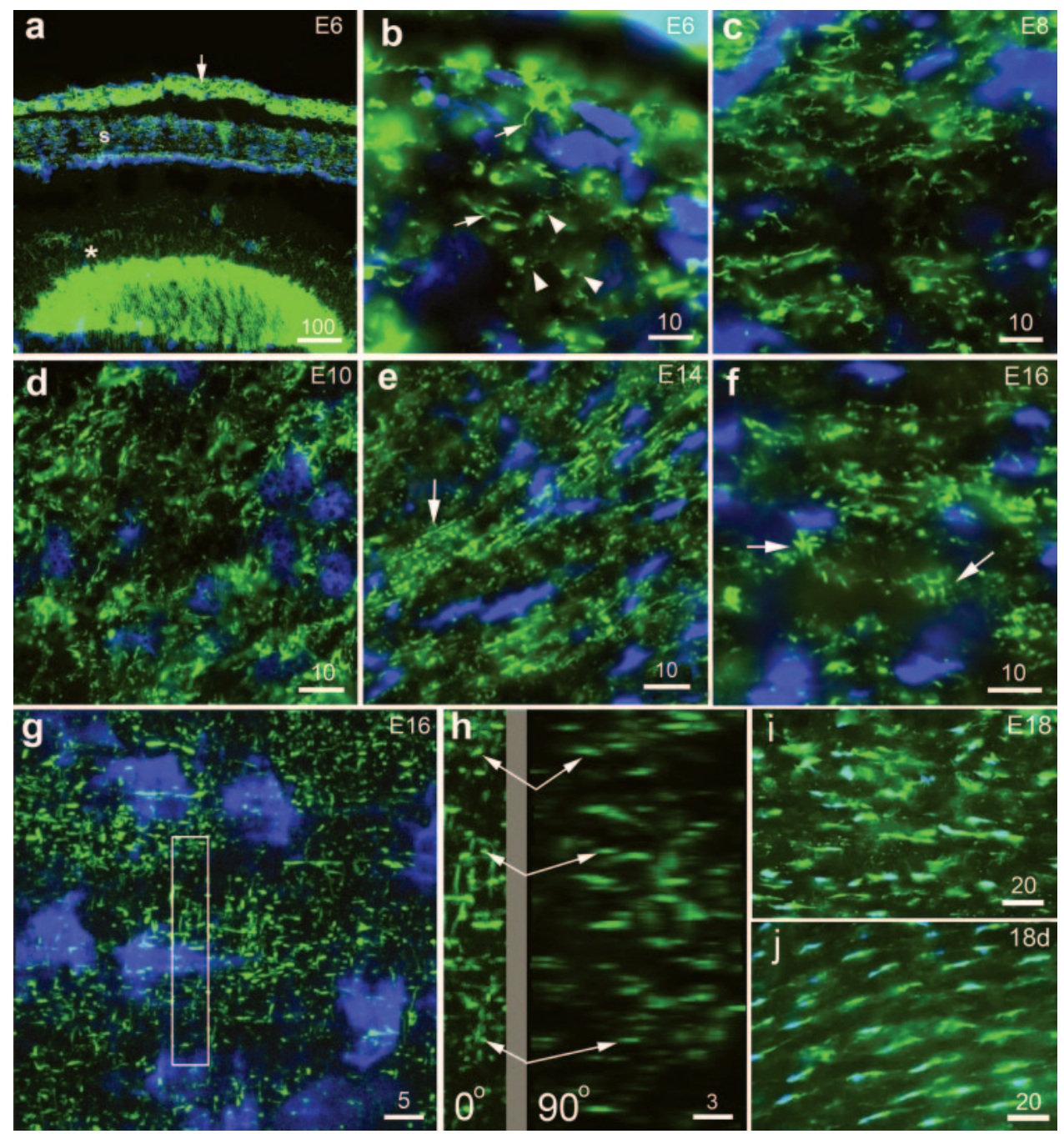

series and the projection rotating about its $Y$-axis are presented as Supplementary Data online at http://www.iovs.org/cgi/content/full/50/4/1653/DC1, in normal and stereoscopic views. (h) Enlargement, and projection rotated through $90^{\circ}$, of the boxed region shown in (g). Arrows link spots of label observed in the face on view with their images in the rotated projection; they form short filaments 2 to $3 \mu \mathrm{m}$ long. An animation of the rotating dataset is present in the Supplementary Data online. (i) Corneal stroma of E18 embryo showing continued presence of actin arrays. (j) Stroma from an 18-day posthatch chicken. Actin label is present with some visible filaments but generally more uniform label of the cell. Scale bar measurements are in microns.

bryonic period studied. Associations were often observed between actin filament bundles and M-38-positive foci, with foci decorating many of the actin filament bundles within the field of view (Fig. 2k, E16).

By comparing SP1.D8 label with DIC images of tangential sections of E10 corneas, it was apparent that the label was in register with collagen fibril bundles in the corneal stroma, suggesting that most, if not all, fibril bundles contain the $\mathrm{N}$-terminus procollagen (Figs. 21-n). High-magnification views of dual labels revealed that the collagen fibril bundles were associated with actin filament bundles running in a similar orientation (Fig. 2o).

\section{Discussion}

Phalloidin labeling has demonstrated an extensive and orthogonal network of prominent actin filament bundles in the developing corneal stroma. At the earliest stages examined here, we speculate that the actin filament bundles could be associated with the invasion of stromal cells into the highly organized primary stroma. ${ }^{15,16}$ Later, the orthogonal array was consistent with the presence of a well-ordered, three-dimensional cellular network accommodating the actin filament bundles. It is possible that filament bundles run in the cell processes that have been observed to extend between keratocytes through the extracellular matrix in adult corneas. ${ }^{28,29}$ Our observations that the orientation of actin filament bundles matches collagen orientation relate to the multiphoton microscopy studies by Svoboda et al., ${ }^{21}$ indicating a close association between collagen orientation and cell orientation, as shown by actin labeling in embryonic chick cornea.

In the posthatch stage, stromal cells were labeled for actin but were without the prominent filament bundles seen in earlier stages. Actin has been reported as having a largely cortical distribution in adult keratocytes. ${ }^{18}$ It could be that the organization we have described is a feature of development associated with cell and matrix orientation and that at later stages we were observing the start of a switch to an adult distribution, when the matrix becomes more fully organized. Similar changes in actin distribution have been observed in the annulus fibrosus of the intervertebral disc, another lamellar fibrous connective tissue. ${ }^{14}$ In the adult cornea, as indicated, label tends to be cortical; however, cells can develop actin fibers in response to injury, with the formation of myofibro- 

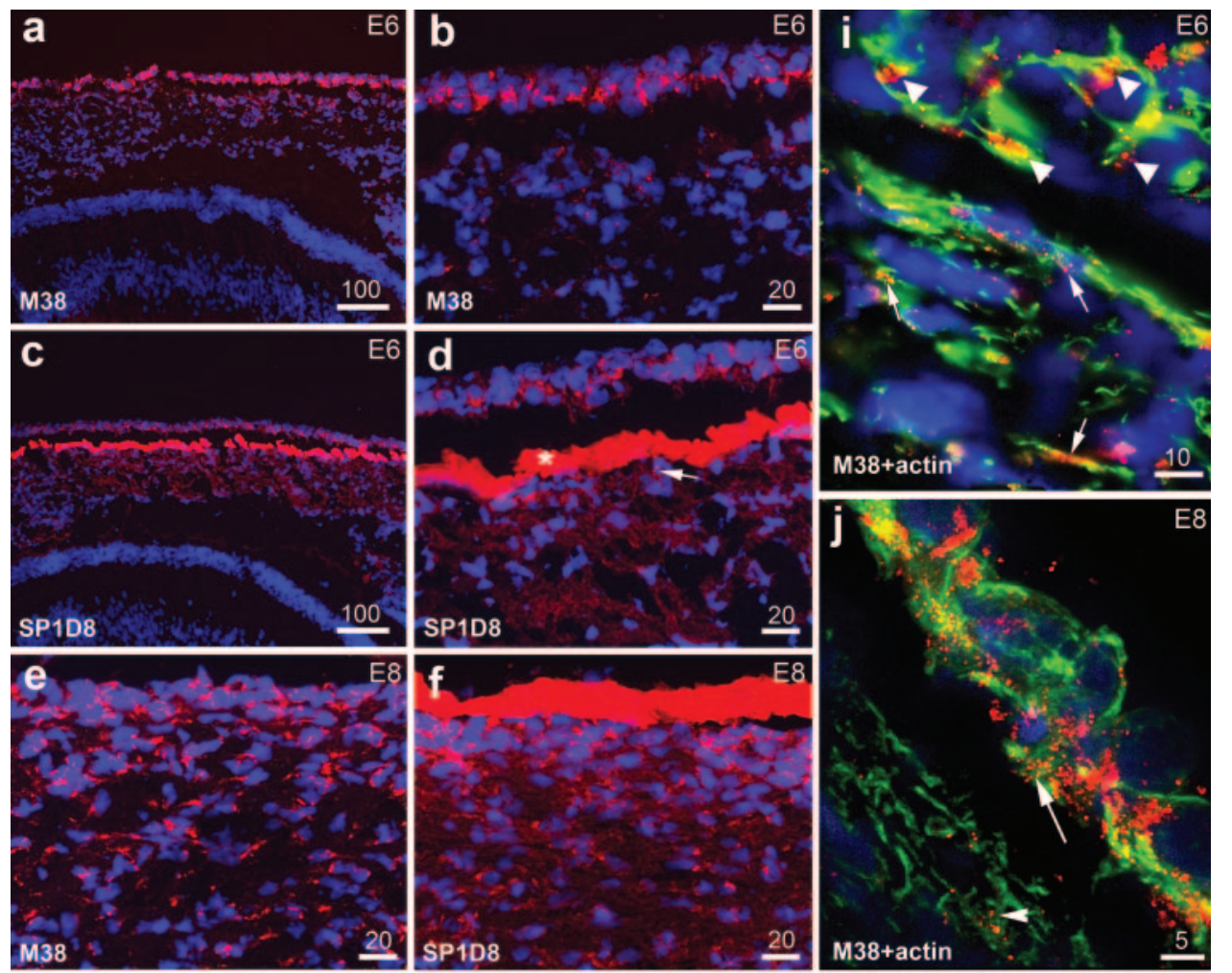

Figure 2. Immunolabels for type I procollagen and dual labels for type I procollagen and actin in developing chicken cornea. Procollagen label is red, actin is green, and nuclei are counterstained blue with DAPI; (j) is a projection derived from confocal section series; the remaining are wide-field fluorescence images. (a, b) Low and high magnification, respectively, of E6 cornea labeled with antibody M-38 to type I collagen Cpropeptide. Label is present intracellularly as small spots in epithelial cells and keratocytes, particularly in the anterior stroma. (c, d) Low and high magnification, respectively, of E6 cornea labeled with antibody SP1.D8 to type I collagen $N$ propeptide. Label is present intracellularly as spots in epithelial cells and can be seen as foci in keratocytes (arrow), though the latter is mostly obscured by positive labeling of the stromal matrix. Bowman's layer (asterisk), from which the epithelium has separated in sectioning, is intensely labeled. (e, f) E8 stroma labeled with M-38 and SP1.D8, respectively. Intracellular foci of label are present in keratocytes with both antibodies in addition the stromal matrix and Bowman's layer label with SP1.D8. (g, h) Dual labels of E16 cornea for procollagens and actin. (g) Extensive distribution of actin filaments in epithelium and stroma and focal labeling for M-38 in the stroma. (h) Label for SP1.D8 distributed throughout the stromal matrix and prominent in Bowman's layer. (i-k) High-magnification views of dual labels for procollagen and actin. (i) Close association of actin networks with $\mathrm{M}-38$ immunolabel in the epithelium (arrowbeads) and stromal fibroblasts (arrows) at E6; (j) confocal projections of section series at E8 show further detail with close association of M-38-positive foci with actin filament bundles in the epithelium (arrow) and anterior stroma (arrowbead), which also shows a high degree of organization of the actin filament bundles. (k) Detailed view of foci of M-38 label apparently positioned on actin filament bundles in keratocytes of the E16 stroma. (1-o) Dual labeling for actin and SP1.D8
matrix label at E10. (1, m) DIC and fluorescence images, respectively; (n) images overlaid. Fibril bundles observed with DIC codistribute with those identified with the SP1.D8 antibody labeling. (o) High-magnification view of the stroma; red-stained collagen fibers have green-labeled actin filaments superimposed on them (circled region). Scale bar measurements are in microns.

blasts during the repair process. ${ }^{17,18}$ It may be that the myofibroblast phenotype, associated with tissue repair and reorientation, is related to that of fibroblastic cells in early development.

We observed actin filament bundles in the embryonic stages running in all three planes relative to the corneal surface-that is, $X$ and $Y$ planes parallel to the anterior surface of the cornea and the $Z$ plane at right angles to this surface. Actin running in the $X$ and $Y$ planes can reasonably be thought of as associated with cells or cell processes running orthogonally and involved with the development of directional, layered, and orthogonal lamellae. ${ }^{7}$ Associations of those running in the $Z$ plane are less clear. It may be that they are simply the same filament bundles but are associated with regions in which layers interweave with one another. ${ }^{30}$ Alternatively, they could have a distinctive function in development. The thickness of the lamellae in the $Z$ plane, 0.5 to $2.5 \mu \mathrm{m}$ in humans ${ }^{30}$ and 2 to $4 \mu \mathrm{m}$ in adult chicken corneas, ${ }^{31}$ is fundamental to corneal structure and function. Perhaps the $Z$ plane filament bundles are involved in controlling this during lamellar deposition.

$\mathrm{N}$ - and $\mathrm{C}$ - propeptides of type I collagen were detected in the epithelium and stroma with the N-propeptide prominent in the extracellular matrix of Bowman's layer and in the rest of the corneal stroma. Epithelial labeling is likely to be associated with the secretion of the collagen of the primary stroma ${ }^{16,25}$ and stromal labeling, with deposition of the secondary stroma. 
Epithelial label is lost at approximately E12, around the time that formation of the primary stroma is coming to an end ${ }^{16}$ and in agreement with in situ hybridization data for collagen gene expression. $^{25}$

Differential distributions of the C- and N-termini of type I procollagen suggest that the $\mathrm{C}$ terminus is cleaved rapidly and degraded on secretion, whereas the N-terminus persists as a component of the extracellular matrix. This supports the previous demonstration of differential rates of processing of the $\mathrm{C}$ and $\mathrm{N}$ - propeptides of type I procollagen in the chick cornea. ${ }^{32}$ Although the propeptides are generally regarded as cleaved and degraded on collagen secretion, a number of other functions have been proposed for them. For example, they provide feedback control by inhibiting the synthesis of collagen RNA in several connective tissue cell types. ${ }^{33-36}$ In developing chick dermis, they are associated with fibrils of different sizes. ${ }^{37}$ Thus, C-propeptides are associated with fibrils covering a range of sizes during development, whereas N-propeptides are associated only with those 20 to $30 \mathrm{~nm}$ in diameter. This is consistent with our observations of N-propeptide distribution in the chick cornea, in which fibrils have diameters of 20 to 30 $\mathrm{nm} .{ }^{38}$ The Bowman's layer labels particularly strongly, and fibrils here are even thinner than in the rest of the cornea. ${ }^{39}$ The N-propeptides also affect tight packing of collagen and may facilitate the fusion of small-diameter fibrils. ${ }^{39}$ It may be that the retention of the collagen type I N-propeptide in the stroma is associated with the formation of fibrils of tightly controlled diameters and spacing, along with other controlling molecules, such as proteoglycans ${ }^{40-43}$ and collagen type $\mathrm{V} .^{38}$

Associations between foci of label for type I procollagen and the actin cytoskeleton were frequently observed, and though no membrane staining was carried out, it is likely that these labels were intracellular in epithelial and stromal cells. Given the resolution constraints on light microscopy, we cannot conclusively state that the two were colocalized, but results were highly suggestive that this was the case. If so, it may be that the actin filament bundles have some involvement in collagen trafficking and secretion. Possibly we were observing secretory vesicles associated with actin filament bundles. In support of this, we point out that there is evidence that actin filament bundles are used for polarized intracellular trafficking in epithelia. ${ }^{44}$

Collagen fibril assembly after secretion is associated with the actin cytoskeleton at the cell surface in several tissues, including cornea. Actin-integrin-fibronectin-collagen interactions have been shown to occur in corneal wound healing, ${ }^{45}$ in keratocytes in culture, ${ }^{46}$ in the developing annulus fibrosus, ${ }^{14}$ and in vascular smooth muscle cells. ${ }^{47}$ It may be that cells also use the actin filaments as a delivery system to points of secretion at the cell surface and for orientation of assembling fibrils, possibly through integrins and other matrix components, after secretion. The recent studies of Canty et al. ${ }^{22-24}$ in tendon extend earlier reports ${ }^{7,48}$ in tendon and cornea to describe mechanisms of deposition of oriented collagen deposition in fibrous connective tissues and the involvement of the actin cytoskeleton in early matrix deposition. Our three-dimensional observations of actin orientation and its possible association with procollagen secretion emphasize the tissue-wide nature of the secretory and orientational mechanisms in producing the stromal matrix.

\section{Acknowledgments}

The authors thank Debbie Tudor for help with production of the E6 and E8 corneal sections.

\section{References}

1. Coulombre AJ, Coulombre JL. The development of the structural and optical properties of the cornea. In Smelser GK, ed. The Structure of the Eye. New York: Academic Press 1961;405-420.

2. Hay ED, Revel J-P. Fine structure of the developing avian cornea. In: Wolsky A, Chen PS, eds. Monographs in Developmental Biology Basel. Geneva: S. Karger; 1969;1:1-144.

3. Trelstad RL, Coulombre AJ. Morphogenesis of the collagenous stroma in the chick cornea. J Cell Biol. 1971;50:840-858.

4. Hay ED. Development of the vertebrate cornea. Int Rev Cytol. 1980;63:263-321.

5. Linsenmayer TF, Fitch JM, Gordon MK, et al. Development and roles of collagenous matrices in the embryonic avian cornea. Prog Retin Eye Res. 1998;17:231-265.

6. Quantock AJ, Young RD. Development of the corneal stroma, and the collagen-proteoglycan associations that help define its structure and function. Dev Dyn. 2008;237:2607-2621.

7. Birk DE, Trelstad RL. Extracellular compartments in matrix morphogenesis: collagen fibril, bundle, and lamellar formation by corneal fibroblasts. J Cell Biol. 1984;99:2024-2033.

8. Linsenmayer TF, Gibney E, Gordon MK, Marchant JK, Hayashi M, Fitch JM. Extracellular matrices of the developing chick retina and cornea: localization of mRNAs for collagen types II and IX by in situ hybridization. Invest Ophtbalmol Vis Sci. 1990;31:1271-1276.

9. Maurice DM. The structure and transparency of the cornea. J Physiol (Lond). 1957;186:263-286.

10. Benedek GB. Theory of transparency of the eye. Appl Optics. 1971;10:459-473.

11. Farrell RA. Corneal transparency. In: Albert DM, Jacobiec SA, eds. Principles and Practices of Opbthalmology. Philadelphia: WB Saunders; 1994.

12. Hickey DS, Hukins DWL. X-ray diffraction studies on the arrangement of collagenous fibres in human foetal intervertebral disc. $J$ Anat. 1980;131:81-90.

13. Humzah MD, Soames RW. Human intervertebral disc: structure and function. Anat Rec. 1988;220:337-356.

14. Hayes AJ, Benjamin M, Ralphs JR. Role of actin stress fibres in the development of the intervertebral disc: cytoskeletal control of extracellular matrix assembly. Dev Dyn. 1999;215:179-189.

15. Bard JBL, Hay ED. The behavior of fibroblasts from the developing avian cornea: morphology and movement in situ and in vitro. J Cell Biol. 1975;67:400-418.

16. Bard JBL, Higginson K. Fibroblast-collagen interactions in the formation of the secondary stroma of the chick cornea. $J$ Cell Biol. 1977;74:816-827.

17. Petroll WM, Cavanagh HD, Barry P, Andrews P, Jester JV. Quantitative analysis of stress fiber orientation during corneal wound healing. J Cell Sci. 1993;104:353-363.

18. Jester JV, Barry PA, Lind GJ, Petroll WM, Garana R, Cavanagh HD. Corneal keratocytes: in situ and in vitro organization of cytoskeletal contractile proteins. Invest Ophthalmol Vis Sci. 1994;35:730 743 .

19. Khoory W, Wu E, Svoboda KKH. Intracellular relationship between actin and alpha-actinin in a whole corneal epithelial tissue. J Cell Sci. 1993;106:703-717.

20. Yeh $\mathrm{B}$, Svoboda KKH. Intracellular distribution of $\beta$-actin mRNA is polarized in embryonic corneal epithelia. J Cell Sci. 1994;107:105115.

21. Svoboda KKH, Petroll MW, Jester JV. Second harmonic signal analysis of whole embryonic avian corneas. Microsc Microanal. 2007; (suppl 2):1550-1551.

22. Canty EG, Lu Y, Meadows RS, Shaw MK, Holmes DF, Kadler KE. Coalignment of plasma membrane channels and protrusions (fibripositors) specifies the parallelism of tendon. J Cell Biol. 2004; 165:553-563.

23. Canty EG, Starborg T, Lu Y, et al. Actin filaments are required for fibripositor-mediated collagen fibril alignment in tendon. $\mathrm{J} \mathrm{Biol}$ Chem. 2006;281:38592-38598.

24. Richardson SH, Starborg T, Lu Y, Humphries SM, Meadows RS, Kadler KE. Tendon development requires regulation of cell condensation and cell shape via cadherin-11-mediated cell-cell junctions. Mol Cell Biol. 2007;27:6218-6228. 
25. Hayashi M, Ninomiya Y, Hayashi K, Linsenmayer TF, Olsen BR Trelstad RL. Secretion of collagen types I and II by epithelial and endothelial cells in the developing chick cornea demonstrated by in situ hybridization and immunohistochemistry. Development. 1988;103:27-36.

26. McDonald JA, Broekelmann TJ, Matheke ML, Crouch E, Koo M, Kuhn C. A monoclonal antibody to the carboxyterminal domain of procollagen type I visualises collagen-synthesizing fibroblasts. J Clin Invest. 1986;78:1237-1244.

27. Foellmer HG, Kawahara K, Madri JA, Furthmayr H, Timpl R, Tuderman L. A monoclonal antibody specific for the amino-terminal cleavage site of procollagen type I. Eur J Biochem. 1983;134:183189.

28. Poole CA, Brookes NH, Clover GM. Keratocyte networks visualised in the living cornea using vital dyes. J Cell Sci. 1993;106:685-691.

29. Poole CA, Brookes NH, Clover GM. Confocal imaging of the human keratocyte network using the vital dye 5-chloromethylfluorescein diacetate. Clin Exp Opbthalmol. 2003;31:147-154.

30. Komai Y, Ushiki T. The three-dimensional organization of collagen fibrils in the human cornea and sclera. Invest Opbthalmol Vis Sci. 1991;32:2244-2258

31. Coulombre AJ. Problems in corneal morphogenesis. Adv Morphol. 1965;4:81-109.

32. Mellor SJ, Atkins GL, Hulmes DJS. Developmental changes in the type I procollagen processing pathway in chick-embryo cornea Biochem J. 1991;276:777-784.

33. Wiestner M, Krieg T, Horlein D, Glanville RW, Fietzek P, Muller PK. Inhibiting effect of procollagen peptides on collagen biosynthesis in fibroblast cultures. J Biol Chem. 1979;254:7016-7023.

34. Perlish JS, Timpl R, Fleischmajer R. Collagen synthesis regulation by the aminopropeptide of procollagen $\mathrm{I}$ in normal and scleroderma fibroblasts. Arthritis Rbeum. 1985;28:647-651.

35. Wu CH, Donavan CB, Wu GY. Evidence for pretranslational regulation of collagen synthesis by procollagen propeptides. $\mathrm{J} \mathrm{Biol}$ Chem. 1986;261:10482-10484.

36. Mizuno M, Fujisawa R, Kuboki Y. The effect of carboxyl-terminal propeptide of type I collagen (c-propeptide) on collagen synthesis of preosteoblasts and osteoblasts. Calcif Tissue Int. 2000;67:391399.
37. Fleischmajer R, Perlish JS, Olsen BR. The carboxylpropeptide of type I procollagen in skin fibrillogenesis. J Invest Dermatol. 1987; 89:212-215.

38. Birk DE, Fitch JM, Linsenmayer TF. Organization of collagen types $\mathrm{I}$ and $\mathrm{V}$ in the embryonic chicken cornea. Invest Opbthalmol Vis Sci. $1986 ; 27: 1470-1477$.

39. Watson RB, Holmes DF, Graham HK, Nusgens BV, Kadler KE. Surface located procollagen N-propeptides on dermatosparactic collagen fibrils are not cleaved by procollagen $\mathrm{N}$-proteinase and do not inhibit binding of decorin to the fibril surface. $\mathrm{J} \mathrm{Mol} \mathrm{Biol}$. 1998;278:195-204

40. Blochberger TC, Vergnes JP, Hempel J, Hassell JR. cDNA to chick lumican (corneal keratan sulphate proteoglycan) reveals homology to the small interstitial proteoglycan gene family and expression in muscle and intestine. J Biol Chem. 1992;267:347-352.

41. Corpuz LM, Funderburgh JL, Funderburgh ML, Bottomley GS, Prakash S, Conrad GW. Molecular cloning and tissue distribution of keratocan: bovine corneal keratan sulphate proteoglycan 37A. J Biol Chem. 1996;271:9759-9763.

42. Funderburgh JL, Corpuz LM, Roth MR, Funderburgh ML, Tasheva ES, Conrad GW. Mimecan, the 25-kDa corneal keratan sulfate proteoglycan, is a product of the gene producing osteoglycin. J Biol Chem. 1997;272:28089-28095.

43. Gealy EC, Kerr BC, Young RD, et al. Differential expression of the keratan sulphate proteoglycan, keratocan, during chick corneal embryogenesis. Histochem Cell Biol. 2007;128:551-555.

44. Rodriguez-Boulan E, Kreitzer G, Müsch A. Organization of vesicular trafficking in epithelia. Nat Rev Mol Cell Biol. 2005;6:233-247.

45. Petroll WM, Cavanagh HD, Jester JV. Assessment of stress fiber orientation during healing of radial keratomy wounds using confocal microscopy. Scanning. 1998;20:74-82.

46. Kim A, Lakshman N, Petroll WM. Quantitative assessment of local collagen matrix remodelling in 3-D culture: the role of Rho kinase. Exp Cell Res. 2006;312:3683-3692.

47. Li S, Van Den Diepstraten C, D'Souza SJ, Chan BMC, Pickering JG. Vascular smooth muscle cells orchestrate the assembly of type I collagen via $\alpha 2 \beta 1$ integrin, RhoA and fibronectin polymerization. Am J Patbol. 2003;163:1045-1056.

48. Birk DE, Trelstad RL. Extracellular compartments in tendon morphogenesis: collagen fibril, bundle and macroaggregate formation. J Cell Biol. 1986;103:231-240. 